\title{
A continuous rainfall model based on vine copulas
}

\author{
H. Vernieuwe ${ }^{1}$, S. Vandenberghe ${ }^{2}$, B. De Baets ${ }^{1}$, and N. E. C. Verhoest ${ }^{2}$ \\ ${ }^{1}$ KERMIT, Department of Mathematical Modelling, Statistics and Bioinformatics, Ghent University, \\ Coupure links 653, 9000 Ghent, Belgium \\ ${ }^{2}$ Laboratory of Hydrology and Water Management, Ghent University, Coupure links 653, 9000 Ghent, Belgium
}

Correspondence to: H. Vernieuwe (hilde.vernieuwe@ugent.be)

Received: 3 November 2014 - Published in Hydrol. Earth Syst. Sci. Discuss.: 14 January 2015

Revised: 20 April 2015 - Accepted: 19 May 2015 - Published: 12 June 2015

\begin{abstract}
Copulas have already proven their flexibility in rainfall modelling. Yet, their use is generally restricted to the description of bivariate dependence. Recently, vine copulas have been introduced, allowing multi-dimensional dependence structures to be described on the basis of a stage by stage mixing of 2-dimensional copulas. This paper explores the use of such vine copulas in order to incorporate all relevant dependences between the storm variables of interest. On the basis of such fitted vine copulas, an external storm structure is modelled. An internal storm structure is superimposed based on Huff curves, such that a continuous time series of rainfall is generated. The performance of the rainfall model is evaluated through a statistical comparison between an ensemble of synthetical rainfall series and the observed rainfall series and through the comparison of the annual maxima.
\end{abstract}

\section{Introduction}

Rainfall serves as an important base for many studies involving hydrological applications including flood risk estimation, the design of hydraulic structure and urban drainage systems or the evaluation of hydrological effects of climate change. Ideally, one should then have extensive observed rainfall time series at hand, both in time and space and at different timescales. Therefore, several rainfall modelling approaches have been proposed during the last decades (e.g. Kavvas and Delleur, 1981; Rodriguez-Iturbe et al., 1987a, b; Katz and Parlange, 1998; Menabde and Sivapalan, 2000; Willems, 2001; Evin and Favre, 2008; Gyasi-Agyei, 2011; Viglione et al., 2012), which can be subdivided into models that generate design storms and models that allow for the simulation of continuous time series at a point or spatially distributed. De- sign storms are generally developed for a given return period and storm duration. The corresponding rainfall volume, obtained from e.g. intensity-duration-frequency (IDF) curves is then assigned to the design storm according to a temporal rainfall pattern or internal storm structure (Chow et al., 1988). However, this approach has an important drawback as it does not properly account for the antecedent wetness state of the catchment (Verhoest et al., 2010). Yet, this initial condition regulates the fractioning of the incident rainfall into runoff and infiltration and thus determines the fluvial response of a catchment to the imposed rainfall event. It was shown by Verhoest et al. (2010) that, because of this, the return period of the rainfall event may differ significantly from that of the corresponding discharge. In order to account for the antecedent soil moisture condition within the catchment, one can alternatively work with continuous rainfall models that provide input to rainfall-runoff models. As the latter models continuously update the soil moisture state, they therefore provide continuous estimates of the antecedent wetness state within the catchment. Continuous rainfall models can be classified into four categories (Onof et al., 2000): (1) physically based meteorological models; (2) stochastic multi-scale models that allow for modelling the spatial evolution of the rainfall process; (3) statistical models, preserving trends in precipitation and (4) stochastic process models that mimic the hierarchical structure of the rainfall process using a limited number of model parameters.

The variables that characterize a storm, i.e. the storm intensity, duration and volume, mostly exhibit some kind of mutual dependence: a long storm duration is more likely to be associated with a low storm intensity than with a high one. It is therefore of utmost importance to construct joint probability distribution functions whenever frequency analy- 
sis studies, e.g. to analyse extremes, need to be carried out. Yet, the marginal probability distribution functions of these storm variables usually do not exhibit the same type of parametric distribution and are largely skewed (Vandenberghe et al., 2010b), i.e. there is a large deviation from the normal distribution. These characteristics hamper the identification of the joint probability distribution functions needed in order to calculate the probability of occurrence of a storm with a specific duration and intensity. The introduction of copulas in hydrology facilitated this task.

Copulas are functions that couple the marginal distribution functions of the random variables into their joint distribution function and therefore describe the dependence structure between these random variables (Sklar, 1959). The great advantage of copulas is that the joint distribution function is built based on two independent tasks comprising the modelling of the dependence and the modelling of the marginal distribution functions. As this property allows for modelling a large variety of joint probability functions, copulas have been used within an increased number of publications in recent years. Pioneering work with respect to applying copulas in hydrology was performed by De Michele and Salvadori (2003), Salvadori and De Michele (2004), Favre et al. (2004) and De Michele et al. (2005). Concerning rainfall modelling, copulas offer a great flexibility in the modelling of high-dimensional dependence structures; however, determining parametric distributions for high-dimensional random vectors is complex (Aas and Berg, 2009). Copulas can, for instance, improve many rainfall models that mimic the external rainfall process, i.e. the process of storm arrival, duration and mean intensity at the coarse scale (Salvadori and De Michele, 2007). These models mostly consider rainfall as a sequence of rectangular pulses, having a certain duration and mean intensity, followed by a specific dry period.

This paper explores how a point-scale rainfall model can be constructed using multivariate copulas, in order to incorporate all relevant dependences between the storm variables of interest. The application of multivariate copulas in hydrology is, in contrast to the application of bivariate copulas, a less explored domain. Some applications can be found in the modelling of trivariate rainfall (Zhang and Singh, 2007; Kao and Govindaraju, 2008; Salvadori and De Michele, 2006; Grimaldi and Serinaldi, 2006), trivariate floods (Serinaldi and Grimaldi, 2007; Genest et al., 2007; Ganguli and Reddy, 2013) and trivariate droughts (Song and Singh, 2010; Wong et al., 2010; Ma et al., 2013). Copula applications in hydrology that go beyond the trivariate case are still very scarce. One example is De Michele et al. (2007) who provide a study on constructing a copula for a 4-dimensional sea storm phenomenon. The lack of successful applications of multivariate copulas in hydrology is, of course, influenced by the progress made in the theory of multivariate copulas. Due to the increase in dimensionality, the study of copulas becomes more complicated than in the bivariate case. Therefore, the question of how to construct a copula family that is suffi- ciently flexible to model the complete dependence structure is a very vivid one in theoretical research. Recently, a flexible construction method, based on mixing (conditional) bivariate copulas, has been introduced, which holds a large potential for many hydrological applications. In literature, this method is referred to as the vine copula (or pair copula) construction method, see e.g. Kurowicka and Cooke (2007), Aas et al. (2009), Aas and Berg (2009) and Hobæk Haff et al. (2010). The underlying theory for this method is given by Bedford and Cooke $(2001,2002)$ and stems from Joe (1997), which also forms the basis for the method of "conditional mixtures", as applied by De Michele et al. (2007). The use of vine copulas is becoming popular in finance (see e.g. Nikololoupoulos et al. (2012); Zhang (2014); Mendes and Accioly (2014)) and geophysics and hydrology (see e.g. Gräler (2014); Xiong et al. (2014); Gyasi-Agyei and Melching (2012); Gräler et al. (2013)).

The model that is developed in this paper consists of two submodels. In a first submodel, the vine copula model, 3and 4-dimensional vine copulas are used to describe the dependence between the storm duration, storm volume, the interstorm period following the storm and, in case a 4dimensional vine copula is used, also the dry fraction within the storm. In a second submodel, the intrastorm-generating model, the intrastorm variability is obtained based on Huff curves (Huff, 1967), which plot the normalized cumulative storm depth against the normalized time since the beginning of a storm. Before introducing the model, Sect. 2 provides some background on the construction of vine copulas and the simulation using vine copulas. Section 3 briefly introduces the historical time series, while Sect. 4 describes the rainfall model. In Sect. 5 the model performance is assessed and a comparison with a state-of-the-art stochastic rainfall model is performed to further assess the performance of the newly introduced model.

\section{Vine copulas}

\subsection{Construction}

A vine copula mixes (conditional) bivariate copulas stage by stage in order to build a high-dimensional copula, i.e. the full density function is decomposed into a product of lowdimensional density functions. Consider the case of two random variables $X$ and $Y$ describing a phenomenon (e.g. storm duration and storm volume). Using their marginal distribution functions $F_{X}$ and $F_{Y}$, the values of both random variables are transformed into values, respectively $U$ and $V$, in the real unit interval $\mathbb{I}=[0,1]$ :

$\left\{\begin{array}{l}u=F_{X}(x) \\ v=F_{Y}(y)\end{array} \Leftrightarrow\left\{\begin{array}{l}x=F_{X}^{-1}(u) \\ y=F_{Y}^{-1}(v),\end{array}\right.\right.$

where $x, y, u$ and $v$ are the values of the corresponding variables $X, Y, U$ and $V$. The $U$ and $V$ are uniformly distributed 
on $\mathbb{I}$. The $F_{X}^{-1}$ and $F_{Y}^{-1}$ are the (quasi-)inverse functions of the distribution functions $F_{X}$ and $F_{Y}$ (Nelsen, 2006).

A bivariate copula or a 2-copula is a function $C: \mathbb{I} \times \mathbb{I} \rightarrow \mathbb{I}$ that satisfies

1. for all $u, v \in \mathbb{I}$,

$$
\begin{array}{lll}
C(u, 0)=0 & \text { and } & C(0, v)=0 \\
C(u, 1)=u & \text { and } & C(1, v)=v,
\end{array}
$$

2. for all $u_{1}, u_{2}, v_{1}, v_{2} \in \mathbb{I}$ for which $u_{1} \leq u_{2}$ and $v_{1} \leq v_{2}$,

$$
C\left(u_{2}, v_{2}\right)-C\left(u_{2}, v_{1}\right)-C\left(u_{1}, v_{2}\right)+C\left(u_{1}, v_{1}\right) \geq 0 .
$$

The extension of this definition to $k$ dimensions results in a $k$-copula (see Nelsen, 2006 for the definition and a detailed explanation). The theorem of Sklar (1959) relates bivariate copulas and bivariate distribution functions and states that for any two continuous random variables $X_{1}$ and $X_{2}$, with continuous marginal cumulative distribution functions $F_{1}$ and $F_{2}$, a unique bivariate copula $C_{12}$ exists such that

$F_{12}\left(x_{1}, x_{2}\right)=C_{12}\left(F_{1}\left(x_{1}\right), F_{2}\left(x_{2}\right)\right)=C_{12}(u, v)$,

where $F_{12}$ is the joint cumulative distribution function of $X_{1}$ and $X_{2}$. This theorem thus formulates that a copula couples the marginal cumulative distribution functions of two random variables into a joint cumulative distribution function $F_{12}$. This theorem can be extended to $k$ dimensions and hence relates a $k$-dimensional cumulative distribution function $F_{12 \ldots k}$ to $k$ marginal distribution functions (Sklar, 1959): for $k$ continuous random variables $X_{1}, X_{2}, \ldots, X_{k}$, with continuous marginal distributions functions $F_{1}, F_{2}, \ldots, F_{k}$, there exists a unique $k$-copula $C_{12 \ldots k}$ such that

$F_{12 \ldots k}\left(x_{1}, x_{2}, \ldots, x_{k}\right)=C_{12 \ldots k}\left(F_{1}\left(x_{1}\right), F_{2}\left(x_{2}\right), \ldots, F_{k}\left(x_{k}\right)\right)$.

In order to explain the construction of vine copulas, the construction of a 3-dimensional vine copula is first explained. The joint probability density function (PDF) $f_{123}$ of a random vector $\left(X_{1}, X_{2}, X_{3}\right)$ can, for instance, be decomposed as follows:

$f_{123}\left(x_{1}, x_{2}, x_{3}\right)=f_{13 \mid 2}\left(x_{1}, x_{3} \mid x_{2}\right) \cdot f_{2}\left(x_{2}\right)$,

where $f_{13 \mid 2}$ is the joint PDF of $X_{1}$ and $X_{3}$, given $X_{2}=x_{2}$ and $f_{2}$ is the marginal PDF of $X_{2}$. The joint cumulative distribution function (CDF) $F_{123}$ is then obtained by integration, following the conditional mixtures approach (De Michele et al.,
2007):

$$
\begin{aligned}
F_{123}\left(x_{1}, x_{2}, x_{3}\right) & =\int_{-\infty}^{x_{1}} \int_{-\infty}^{x_{2}} \int_{-\infty}^{x_{3}} f_{123}(r, s, t) \mathrm{d} r \mathrm{~d} s \mathrm{~d} t \\
& =\int_{-\infty}^{x_{1}} \int_{-\infty}^{x_{2}} \int_{-\infty}^{x_{3}} f_{13 \mid 2}(r, t \mid s) f_{2}(s) \mathrm{d} r \mathrm{~d} s \mathrm{~d} t \\
& =\int_{-\infty}^{x_{2}}\left[\int_{-\infty}^{x_{1}} \int_{-\infty}^{x_{3}} f_{13 \mid 2}(r, t \mid s) \mathrm{d} r \mathrm{~d} t\right] f_{2}(s) \mathrm{d} s \\
& =\int_{-\infty}^{x_{2}} F_{13 \mid 2}\left(x_{1}, x_{3} \mid s\right) \mathrm{d} F_{2}(s) \\
& =\int_{-\infty}^{x_{2}} C_{13 \mid 2}\left(F_{1 \mid 2}\left(x_{1} \mid s\right), F_{3 \mid 2}\left(x_{3} \mid s\right)\right) \mathrm{d} F_{2}(s)
\end{aligned}
$$

The conditional CDFs $F_{1 \mid 2}\left(x_{1} \mid x_{2}\right)$ and $F_{3 \mid 2}\left(x_{3} \mid x_{2}\right)$ can also be expressed in terms of copulas:

$$
\begin{aligned}
& F_{1 \mid 2}\left(x_{1} \mid x_{2}\right)=\frac{\partial}{\partial u_{2}} C_{12}\left(u_{1}, u_{2}\right) \\
& F_{3 \mid 2}\left(x_{3} \mid x_{2}\right)=\frac{\partial}{\partial u_{2}} C_{23}\left(u_{2}, u_{3}\right),
\end{aligned}
$$

with $u_{1}=F_{1}\left(x_{1}\right), u_{2}=F_{2}\left(x_{2}\right)$ and $u_{3}=F_{3}\left(x_{3}\right)$. When instead of $X_{1}, X_{2}$ and $X_{3}$, their transformed uniform random variables on $\mathbb{I}$, namely $U_{1}, U_{2}$ and $U_{3}$, are considered, Eq. (7) can be expressed as follows:

$C_{123}=\int_{0}^{u_{2}} C_{13 \mid 2}\left(\frac{\partial}{\partial s} C_{12}\left(u_{1}, s\right), \frac{\partial}{\partial s} C_{23}\left(s, u_{3}\right)\right) \mathrm{d} s$.

In the theory of vine copulas, the same decomposition of the density function is performed, but instead of using cumulative probability functions, all equations are rather expressed in terms of density functions and the full density function $c_{123}$ of the 3 -dimensional copula is then given by

$$
\begin{aligned}
& c_{123}\left(u_{1}, u_{2}, u_{3}\right)=c_{13 \mid 2}\left(F_{1 \mid 2}\left(x_{1} \mid x_{2}\right), F_{3 \mid 2}\left(x_{3} \mid x_{2}\right)\right) \\
& \cdot c_{12}\left(u_{1}, u_{2}\right) \cdot c_{23}\left(u_{2}, u_{3}\right) .
\end{aligned}
$$

Similarly, for a random vector $\left(X_{1}, X_{2}, X_{3}, X_{4}\right)$, the joint PDF $f_{1234}$ can, for instance, be decomposed as follows:

$f_{1234}\left(x_{1}, x_{2}, x_{3}, x_{4}\right)=f_{14 \mid 23}\left(x_{1}, x_{4} \mid x_{2}, x_{3}\right) \cdot f_{23}\left(x_{2}, x_{3}\right)$. 


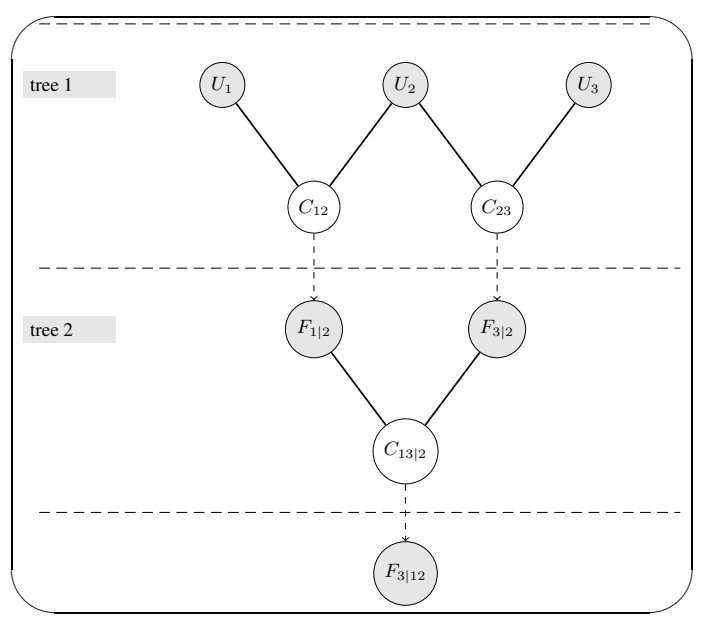

Figure 1. Hierarchical nesting of bivariate copulas in the construction of a 3-dimensional vine copula through conditional mixtures.

The joint cumulative distribution function $F_{1234}$ is then obtained by integration, similarly as in Eq. (7):

$F_{1234}\left(x_{1}, x_{2}, x_{3}, x_{4}\right)=\int_{-\infty}^{x_{2}} \int_{-\infty}^{x_{3}} C_{14 \mid 23}\left(F_{1 \mid 23}\left(x_{1} \mid s, t\right)\right.$,

$\left.F_{4 \mid 23}\left(x_{4} \mid s, t\right)\right) \mathrm{d} F_{23}(s, t)$.

Herein, the derivative of the bivariate $\operatorname{CDF} F_{23}\left(x_{2}, x_{3}\right)$ is expressed as $\mathrm{d} F_{23}\left(x_{2}, x_{3}\right)=f_{23}\left(x_{2}, x_{3}\right) \mathrm{d} x_{2} \mathrm{~d} x_{3}$. The functions $F_{1 \mid 23}\left(x_{1} \mid x_{2}, x_{3}\right)$ and $F_{4 \mid 23}\left(x_{4} \mid x_{2}, x_{3}\right)$ are conditional CDFs (or CCDFs), and can also be expressed in terms of copulas:

$F_{1 \mid 23}\left(x_{1} \mid x_{2}, x_{3}\right)=\frac{\partial C_{12 \mid 3}\left(F_{1 \mid 3}\left(x_{1} \mid x_{3}\right), F_{2 \mid 3}\left(x_{2} \mid x_{3}\right)\right)}{\partial F_{2 \mid 3}\left(x_{2} \mid x_{3}\right)}$
$F_{4 \mid 23}\left(x_{4} \mid x_{2}, x_{3}\right)=\frac{\partial C_{24 \mid 3}\left(F_{2 \mid 3}\left(x_{2} \mid x_{3}\right), F_{4 \mid 3}\left(x_{4} \mid x_{3}\right)\right)}{\partial F_{2 \mid 3}\left(x_{2} \mid x_{3}\right)}$

where the CCDFs $F_{1 \mid 3}, F_{2 \mid 3}$ and $F_{4 \mid 3}$ are calculated as in Eq. (8).

\subsection{Fitting a 3- or 4-dimensional vine copula}

Figures 1 and 2 illustrate the principle of constructing a 3respectively 4-dimensional vine copula. Consider tree 1 in Figs. 1 and 2 where three (respectively four) uniform (on $[0,1]$ ) random variables $U_{1}, U_{2}$ and $U_{3}$ (or $U_{1}, U_{2}, U_{3}$ and $U_{4}$ ) are given and their pairwise dependences are described by the bivariate copulas $C_{12}$ and $C_{23}$ (respectively $C_{12}, C_{23}$ and $C_{34}$ ). Given a specific value of the second variable, these bivariate copulas can be conditioned (cf. dashed arrows in Figs. 1 and 2) through partial differentiation (Aas et al., 2009), resulting in the CCDFs $F_{1 \mid 2}$ and $F_{3 \mid 2}$ (respectively $F_{1 \mid 2}, F_{3 \mid 2}, F_{2 \mid 3}$ and $F_{4 \mid 3}$ ). The pairwise dependences between these CCDFs are then captured by the bivariate copula $C_{13 \mid 2}$ (respectively the copulas $C_{13 \mid 2}$ and $C_{24 \mid 3}$ ). See tree

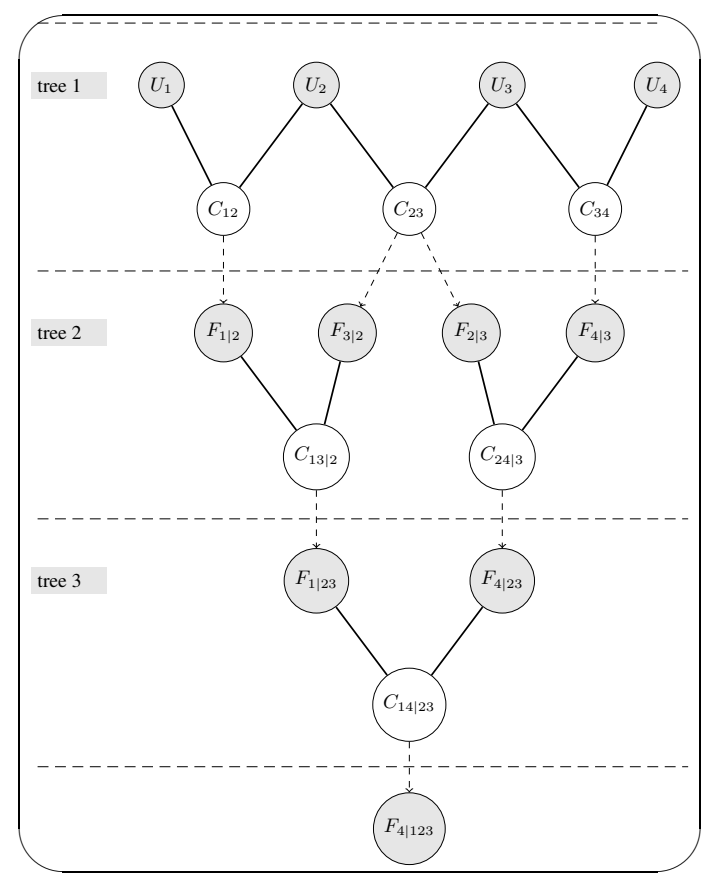

Figure 2. Hierarchical nesting of bivariate copulas in the construction of a 4-dimensional vine copula through conditional mixtures.

2 in Figs. 1 and 2. These latter copulas can then also be conditioned by partial differentiation to obtain $F_{3 \mid 12}$ (respectively $F_{3 \mid 12}$ and $F_{4 \mid 23}$ ). For the 4-dimensional vine copula, another bivariate copula $C_{14 \mid 23}$ captures the pairwise dependence between these CCDFs and can on its turn be partially differentiated to obtain $F_{4 \mid 123}$. See tree 3 in Figure 2. The conditional CDFs $F_{3 \mid 12}$ and $F_{4 \mid 123}$ (of the 3- and 4-dimensional vine copula, respectively) will be of use for simulation purposes (Aas et al., 2009). It should be noted that the hierarchical nesting of bivariate (conditional) copulas as presented here is just one of the possibilities and corresponds to what is called a D-vine (Aas et al., 2009).

In practice, the bivariate copulas in a higher tree of the vine copula (e.g. $C_{13 \mid 2}$ ) are fitted as follows. Consider a set of $n$ data points, for all triplets $\left(u_{1, i}, u_{2, i}, u_{3, i}\right)$ (or for all quadruplets $\left.\left(u_{1, i}, u_{2, i}, u_{3, i}, u_{4, i}\right)\right), i=1, \ldots, n$, the CCDF values (e.g. the CDF values according to $F_{1 \mid 2}$ and $F_{3 \mid 2}$ in the case of $C_{13 \mid 2}$ ) are calculated. The bivariate copulas (e.g. $C_{13 \mid 2}$ ) are then fitted to these "conditioned observations", which are again approximately uniformly distributed on $\mathbb{I}$.

\subsection{Generating samples out of the vine copula}

A general simulation algorithm is presented next, borrowed from the theory on conditional mixtures. The literature on vine copulas reports very similar simulation algorithms (Aas and Berg, 2009; Aas et al., 2009). In order to simulate a random sample $\left(u_{1}, u_{2}, u_{3}\right)$ (or $\left.\left(u_{1}, u_{2}, u_{3}, u_{4}\right)\right)$ out of the 3-D (or 4-D) conditional mixture copula, i.e. $U_{1}, U_{2}, U_{3}$ (and $U_{4}$ ) 
are uniformly distributed on $\mathbb{I}$, the 1-dimensional CCDF is highly important and is defined as (De Michele et al., 2007)

$$
\begin{aligned}
& G_{k \mid 1 \ldots k-1}\left(u_{k} \mid u_{1}, \ldots, u_{k-1}\right) \\
& =\mathbb{P}\left(U_{k} \leq u_{k} \mid U_{1}=u_{1}, \ldots, U_{k-1}=u_{k-1}\right) \\
& =\frac{\frac{\partial^{k-1}}{\partial u_{1} \ldots \partial u_{k-1}} C_{1 \ldots k}\left(u_{1}, \ldots, u_{k}\right)}{\frac{\partial^{k-1}}{\partial u_{1} \ldots \partial u_{k-1}} C_{1 \ldots k-1}\left(u_{1}, \ldots, u_{k-1}\right)}
\end{aligned}
$$

Herein, the numerator is the mixed partial derivative of the $k$-dimensional copula with respect to the conditioning variables. The denominator is the copula density of the $(k-1)$ dimensional copula of the conditioning variables. In order to simulate a random sample out of the 3-D (respectively 4D) conditional mixture copula, a random sample $\left(t_{1}, t_{2}, t_{3}\right)$ (or $\left.t_{1}, t_{2}, t_{3}, t_{4}\right)$ should be first generated from $\left(T_{1}, T_{2}, T_{3}\right)$ (respectively $T_{1}, T_{2}, T_{3}, T_{4}$ ) which are uniformly distributed random variables on $\mathbb{I}$, and serve as random probability levels of the CCDFs in the simulation algorithm which is listed next (of course for generating a 3-dimensional sample, step 4 should not be performed):

1. $u_{1}=t_{1}$;

2. $u_{2}=G_{2 \mid 1}^{-1}\left(t_{2} \mid u_{1}\right)$, where

$$
G_{2 \mid 1}\left(u_{2} \mid u_{1}\right)=\frac{\partial}{\partial u_{1}} C_{12}\left(u_{1}, u_{2}\right)
$$

3. $u_{3}=G_{3 \mid 12}^{-1}\left(t_{3} \mid u_{1}, u_{2}\right)$, where

$$
G_{3 \mid 12}\left(u_{3} \mid u_{1}, u_{2}\right)=\frac{\frac{\partial^{2}}{\partial u_{1} \partial u_{2}} C_{123}\left(u_{1}, u_{2}, u_{3}\right)}{\frac{\partial^{2}}{\partial u_{1} \partial u_{2}} C_{12}\left(u_{1}, u_{2}\right)} ;
$$

4. $u_{4}=G_{4 \mid 123}^{-1}\left(t_{4} \mid u_{1}, u_{2}, u_{3}\right)$, where

$$
\begin{aligned}
& G_{4 \mid 123}\left(u_{4} \mid u_{1}, u_{2}, u_{3}\right) \\
& =\frac{\frac{\partial^{3}}{\partial u_{1} \partial u_{2} \partial u_{3}} C_{1234}\left(u_{1}, u_{2}, u_{3}, u_{4}\right)}{\frac{\partial^{3}}{\partial u_{1} \partial u_{2} \partial u_{3}} C_{123}\left(u_{1}, u_{2}, u_{3}\right)} .
\end{aligned}
$$

The calculation of some partial derivatives, necessary for obtaining the $\mathrm{CCDF} G_{4 \mid 123}$ is given below:

$$
\begin{aligned}
& \frac{\partial^{3}}{\partial u_{1} \partial u_{2} \partial u_{3}} C_{1234}\left(u_{1}, u_{2}, u_{3}, u_{4}\right) \\
& =\frac{\partial}{\partial u_{1}} C_{14 \mid 23}\left(G_{1 \mid 23}\left(u_{1} \mid u_{2}, u_{3}\right), G_{4 \mid 23}\left(u_{4} \mid u_{2}, u_{3}\right)\right),
\end{aligned}
$$

with

$$
\begin{aligned}
& G_{1 \mid 23}\left(u_{1} \mid u_{2}, u_{3}\right)=\frac{\frac{\partial^{2}}{\partial u_{2} \partial u_{3}} C_{123}\left(u_{1}, u_{2}, u_{3}\right)}{\frac{\partial^{2}}{\partial u_{2} \partial u_{3}} C_{23}\left(u_{2}, u_{3}\right)} \\
& =\frac{\frac{\partial}{\partial u_{3}} C_{13 \mid 2}\left(\frac{\partial}{\partial u_{2}} C_{12}\left(u_{1}, u_{2}\right), \frac{\partial}{\partial u_{2}} C_{23}\left(u_{2}, u_{3}\right)\right)}{\frac{\partial^{2}}{\partial u_{2} \partial u_{3}} C_{23}\left(u_{2}, u_{3}\right)},
\end{aligned}
$$

and

$$
\begin{gathered}
G_{4 \mid 23}\left(u_{4} \mid u_{2}, u_{3}\right)=\frac{\frac{\partial^{2}}{\partial u_{2} \partial u_{3}} C_{234}\left(u_{2}, u_{3}, u_{4}\right)}{\frac{\partial^{2}}{\partial u_{2} \partial u_{3}} C_{23}\left(u_{2}, u_{3}\right)} \\
=\frac{\frac{\partial}{\partial u_{3}} C_{24 \mid 3}\left(\frac{\partial}{\partial u_{3}} C_{23}\left(u_{2}, u_{3}\right), \frac{\partial}{\partial u_{3}} C_{34}\left(u_{3}, u_{4}\right)\right)}{\frac{\partial^{2}}{\partial u_{2} \partial u_{3}} C_{23}\left(u_{2}, u_{3}\right)} .
\end{gathered}
$$

Once $u_{1}, u_{2}, u_{3}$ and $u_{4}$ are simulated, the corresponding values of $x_{1}, x_{2}, x_{3}$ and $x_{4}$ can be calculated by means of the inverse marginal CDFs $F_{1}^{-1}, F_{2}^{-1}, F_{3}^{-1}$ and $F_{4}^{-1}$, respectively.

\section{Historical time series characteristics}

The time series used in this paper for fitting the model consists of a 105-year 10 min rainfall record of Uccle, Belgium. These data were obtained by a Hellmann-Fuess pluviograph, installed in and operated by the Royal Meteorological Institute at Uccle near Brussels, Belgium (Demarée, 2003). This exceptional time series has been used in several studies, albeit with varying lengths, concerning statistical analyses (Vaes et al., 2002; De Jongh et al., 2006; Ntegeka and Willems, 2008; Vandenberghe et al., 2010b) and stochastic rainfall modelling (Verhoest et al., 1997; Vandenberghe et al., 2011; Vanhaute et al., 2012; Evin and Favre, 2013; Pham et al., 2013). For the current study, storms were first selected and their characteristics of interest calculated. Storms were selected on the basis of a minimal dry duration that separates two storms. Any dry period shorter than this threshold is thus considered to be part of a storm (Bonta and Rao, 1988). Similar to Verhoest et al. (1997) and Vandenberghe et al. (2010a, b, 2011), a dry period of $24 \mathrm{~h}$ was chosen, as this period assures that the arrival times of independent storms are Poisson distributed (Restrepo-Posada and Eagleson, 1982). In this way, 8665 storms were selected and the following characteristics calculated: the onset of a storm (day, month, year), the storm volume $V(\mathrm{~mm})$, the storm duration $W(\mathrm{~h})$, the dry duration after the storm $D(\mathrm{~h})$, and the fraction dry 
Table 1. Observed probability of $p_{\mathrm{d}}=0$ for the storms in the different seasons

\begin{tabular}{lr}
\hline & $\begin{array}{r}\text { Probability } \\
\text { of } p_{\mathrm{d}}=0\end{array}$ \\
\hline Winter & $8.75 \%$ \\
Spring & $11.21 \%$ \\
Summer & $13.13 \%$ \\
Autumn & $12.14 \%$ \\
\hline
\end{tabular}

within the storm $p_{\mathrm{d}}$. It was observed that events with identical values of variables occur in the observed time series. As this is not desirable for a copula-based analysis, in which ranks are important, random noise, uniformly distributed between -0.1 and $+0.1 \mathrm{~mm}$ was introduced to all strictly positive 10 min observations as described and motivated in Vandenberghe et al. (2010b). The choice of $0.1 \mathrm{~mm}$ was based on the pluviograph's resolution. We also refer to Vandenberghe et al. (2010b) for a profound analysis of the dependences between the variables $W, V$, and $D$. Vandenberghe et al. (2010b) asserted that the hypothesis of stationarity of storms on the Uccle time series is fulfilled through seasonally subdividing the storms allowing copulas to be fitted per season. Therefore, the storm characteristics of the 105 -year $10 \mathrm{~min}$ rainfall time series are subdivided according to the season in which the storms occurred. To this end, similarly as in Vandenberghe et al. (2010b), winter is defined as the months December, January and February, spring as the months March, April and May, summer as the months June, July and August and autumn as the months September, October and November. It was furthermore noticed that some of the storms have no internal dry $10 \mathrm{~m}$ intervals, i.e. $p_{\mathrm{d}}=0$. The set of storms within each season is then further subdivided into a subset of storms for which $p_{\mathrm{d}}=0$ and a subset of storms for which $p_{\mathrm{d}} \neq 0$. The observed probability of $p_{\mathrm{d}}=0$ for the different seasons is listed in Table 1.

A kernel-smoothed distribution function was fitted to the observed values of $W, V, D$ and $p_{\mathrm{d}}$ as none of the commonly used probability distributions fitted the data well. As $D$ has a theoretical minimum of $24 \mathrm{~h}$, due to the selection criterion, the distribution was fit to $D-24 \mathrm{~h}$ values, and afterwards, $24 \mathrm{~h}$ were added.

As the storm characteristics $V, W$ and $D$ do not reveal any information on the internal storm structure, and $p_{\mathrm{d}}$ only gives partial information, Huff curves, as derived in Vandenberghe et al. (2010a), are employed to provide statistical information on the internal structure. The idea to use Huff curves to generate an internal storm structure has also been adopted by Candela et al. (2014). Given the 105-year time series at hand, empirical Huff curves can be obtained by partitioning each storm in the time series in e.g. 20 identical time intervals at every $5 \%$ of the total storm duration. Furthermore, storms were classified into seasons, and quartile groups according

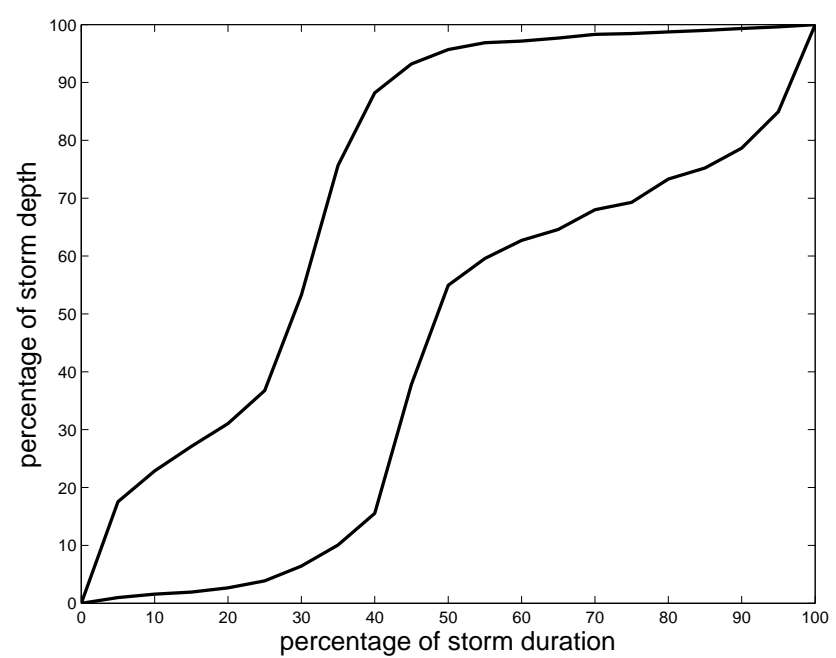

Figure 3. Huff curves for the second-quartile autumn storms. The $10 \%$ (lower) and $90 \%$ (upper) percentile curves are given.

to the quarter of the storm duration that received the largest amount of rainfall. For each season and each quartile group the corresponding Huff curves were obtained by visualizing the 10 and $90 \%$ percentiles of the distribution. In this way, 16 Huff curves were obtained (four quartile groups per season). As an example, Fig. 3 illustrates the 10 and $90 \%$ percentile curves of the second-quartile autumn storms. Vandenberghe et al. (2010a) showed that these curves are independent of the extremity of the storm.

\section{Description of the rainfall model}

\subsection{The vine copula submodel: construction and use of vine copulas in the generation of a time series}

By examining the storm characteristics of the historical time series, it is observed that some storms have internal dry $10 \mathrm{~min}$ intervals while others have not. It was decided to fit, for each season, a 4-dimensional vine copula to the values of $W, V, D$ and the non-zero values of $p_{\mathrm{d}}$. Furthermore, for each season, a 3-dimensional vine copula was fitted to the values of $W, V$ and $D$ in the case $p_{\mathrm{d}}=0$. In this way, dependences between the variables for $p_{\mathrm{d}}=0$ and $p_{\mathrm{d}} \neq 0$ are taken into account and four 3-dimensional and four 4-dimensional vine copulas are obtained.

The ordering of the copulas in the vine copula, i.e. the selection of a D-vine, is based on the values of Kendall's tau as listed in Table 2. These values show that the strongest dependences exist between the variables $W$ and $V, p_{\mathrm{d}}$ and $W$, and $V$ and $D$. By putting the most dependent pair of variables in the first tree of the vine copula, the structure of a Dvine is established. The 3- ( $W, V$, and $D)$ and 4-dimensional $\left(p_{\mathrm{d}}, W, V, D\right)$ vine copulas are fitted stage by stage, following the method explained in Sect. 2.2. We are aware that 

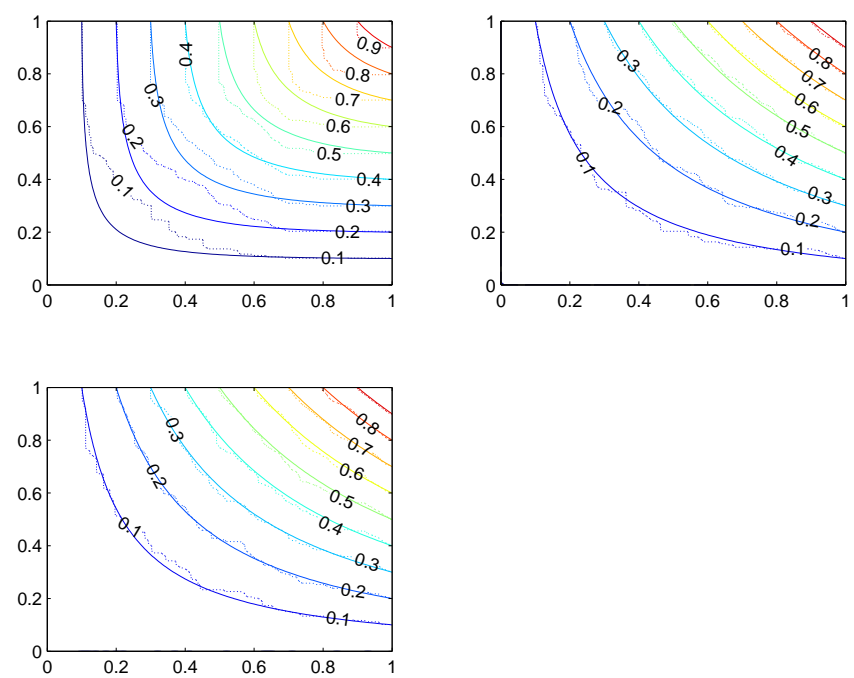

Figure 4. Contour plots of the empirical (dotted lines) and the fitted Frank copulas (solid lines) for the different trees in the 3dimensional vine copula for season 1 . Bivariate copulas between $W$ and $V$ and $V$ and $D$ (top panel) and between $W \mid V$ and $D \mid V$ (bottom panel) are shown.

different copula families could be used to describe the dependences between the different variables (see Vandenberghe et al., 2010b). Yet, in this conceptual study, we opted to restrict to the Frank copula family to describe the (conditional) bivariate dependences within the vine copulas, because of its ability to represent positive or negative dependence. Furthermore, this family is frequently applied to describe bivariate hydrological phenomena (Pan et al., 2013). Alternative families could better fit the different dependences within the vine copula - however, the search for the best fitting copula was out of the scope of the current study. It should be remarked that two different ways of parametrizing the Frank copula exist. In this paper, the one that has the dependence parameter range of $[-\infty,+\infty]$ is employed. The parameters of the Frank copulas are numerically estimated using the relationship between Kendall's tau and the parameter value of the Frank copula (Genest, 1987):

$\tau_{K}=1-\frac{4}{\theta}\left(1-\frac{1}{\theta} \int_{0}^{\theta} \frac{t}{e^{t}-1} d t\right)$.

The fitted Frank copula parameters are presented in Table 3. Figures 4 and 5 show the contours of the Frank copulas and the empirical copulas for the 3- and 4-dimensional vine copula for the first season. It can be seen that the Frank copula fits the empirical copulas fairly well. Only the dependence between $W$ and $V$ in the 3-dimensional vine copula and the dependence between $p_{\mathrm{d}}$ and $W$, and $W \mid V$ and $D \mid V$ in the 4-dimensional vine copula are less well represented. In order to check whether the vine copulas preserve the dependence between the variables, two samples, with size 10000 ,
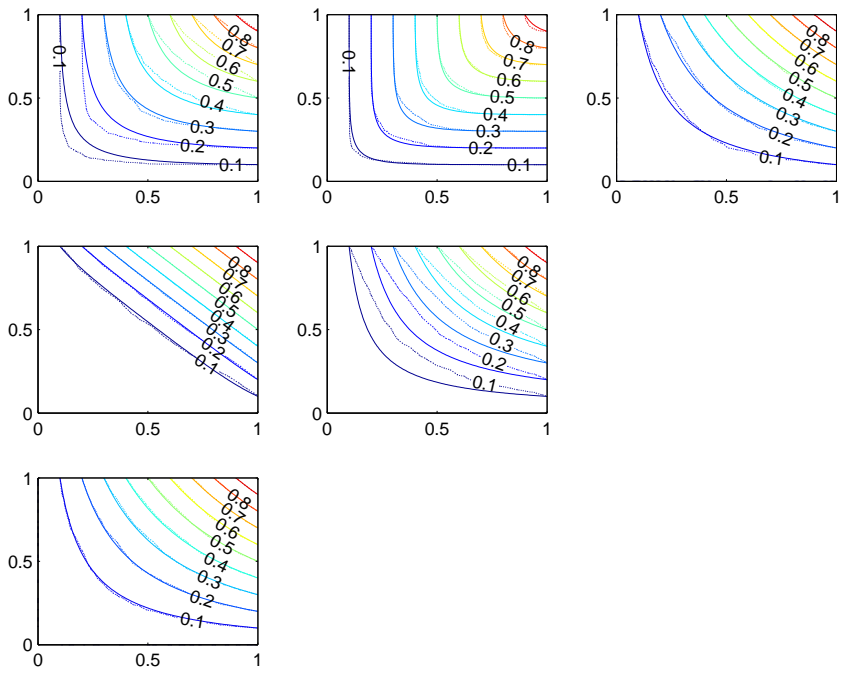

Figure 5. Contour plots of the empirical (dotted lines) and the fitted Frank copulas (solid lines) for the different trees in the 4dimensional vine copula for season 1 . Bivariate copulas between $p_{\mathrm{d}}$ and $W, W$ and $V$, and $V$ and $D$ (top panel), between $p_{\mathrm{d}} \mid W$ and $V \mid W$, and $W \mid V$ and $D \mid V$ (middle panel) and between $p_{\mathrm{d}} \mid W V$ and $D \mid W V$ (bottom panel) are shown.

are simulated using the 3- and 4-dimensional vine copulas, respectively, based on the method described in Sect. 2.3. Table 2 shows the good correspondence between the observed and simulated pairwise dependences. To be able to transform samples from the fitted copula to real samples, the (inverse) marginal cumulative distribution functions of $p_{\mathrm{d}}, W, V$ and $D$ are employed.

The inverse CDFs are then used to transform simulated uniformly distributed values in $\mathbb{I}$ to values in $\mathbb{R}$. Once the simulated values of the quadruplet $\left(p_{\mathrm{d}}, W, V, D\right)$ are known, a time series of 105 years of rectangular rainfall pulses with duration $W$ and height $I=V / W$, being separated by a dry duration $D$, is obtained. Of course, these rectangular pulses only correspond to the external storm structure. At this stage, only the timing of the beginning and the end of a storm is important. The pulse itself, characterized by $W, V$ and $p_{\mathrm{d}}$, needs to be further disaggregated into finer-scale rainfall, which is elaborated upon in the next section.

\subsection{The intrastorm-generating submodel: disaggregation of rectangular pulses by means of Huff curves}

In order to employ Huff curves in the disaggregation of the rectangular pulses, a random quartile group is first assigned based on the probabilities of occurrence of the four quartile groups, as indicated in Table 4. By assigning the first pulse of the generated time series to the beginning of the year, the season of each simulated pulse is easily derived. On the basis of the probability of $p_{\mathrm{d}}=0$, the 3- or 4-dimensional vine copula for the corresponding season is employed to obtain 
Table 2. Correspondence between the observed and simulated pairwise dependences among $\left(p_{\mathrm{d}}, W, V, D\right)$, expressed as Kendall's tau $\tau_{K}$.

\begin{tabular}{|c|c|c|c|c|c|}
\hline & & Observed 3-D & Observed 4-D & Simulated 3-D & Simulated 4-D \\
\hline \multirow{6}{*}{ Season 1} & $\tau_{12}$ & - & 0.3040 & - & 0.3066 \\
\hline & $\tau_{23}$ & 0.4140 & 0.6361 & 0.4118 & 0.6305 \\
\hline & $\tau_{34}$ & -0.0804 & -0.0736 & -0.0754 & -0.0790 \\
\hline & $\tau_{13}$ & - & -0.0336 & - & -0.0092 \\
\hline & $\tau_{24}$ & -0.0831 & -0.0341 & -0.0760 & -0.0425 \\
\hline & $\tau_{14}$ & - & 0.0259 & - & 0.0111 \\
\hline \multirow{6}{*}{ Season 2} & $\tau_{12}$ & - & 0.3418 & - & 0.3423 \\
\hline & $\tau_{23}$ & 0.5318 & 0.5888 & 0.5314 & 0.5866 \\
\hline & $\tau_{34}$ & -0.0773 & -0.0778 & -0.0811 & -0.0649 \\
\hline & $\tau_{13}$ & - & -0.0183 & - & -0.0018 \\
\hline & $\tau_{24}$ & -0.0397 & -0.0410 & -0.0421 & -0.0416 \\
\hline & $\tau_{14}$ & - & 0.0221 & - & 0.0026 \\
\hline \multirow{6}{*}{ Season 3} & $\tau_{12}$ & - & 0.4060 & - & 0.4132 \\
\hline & $\tau_{23}$ & 0.5243 & 0.5540 & 0.5248 & 0.5483 \\
\hline & $\tau_{34}$ & 0.0247 & -0.0855 & 0.0222 & -0.0820 \\
\hline & $\tau_{13}$ & - & 0.0411 & - & 0.0602 \\
\hline & $\tau_{24}$ & -0.0079 & -0.0613 & -0.0109 & -0.0672 \\
\hline & $\tau_{14}$ & - & -0.0126 & - & -0.0332 \\
\hline \multirow{6}{*}{ Season 4} & $\tau_{12}$ & - & 0.3208 & - & 0.3276 \\
\hline & $\tau_{23}$ & 0.4887 & 0.6058 & 0.4948 & 0.6016 \\
\hline & $\tau_{34}$ & -0.1066 & -0.0779 & -0.1084 & -0.0816 \\
\hline & $\tau_{13}$ & - & -0.0287 & - & -0.0080 \\
\hline & $\tau_{24}$ & -0.1377 & -0.0636 & -0.1680 & -0.0737 \\
\hline & $\tau_{14}$ & - & -0.0086 & - & -0.0099 \\
\hline
\end{tabular}

simulated storm pulses. Next, as the season and the quartile group are known, a random internal storm structure can be assigned to each simulated storm pulse on the basis of the corresponding 10 and $90 \%$ Huff curves. To this end, time instants corresponding to the end of each 10 min interval within the storm are selected. The 10 and $90 \%$ curves are then interpolated such that the values of the normalized cumulative storm depth for each of these time instants (expressed as a percentage of the total storm duration) are obtained.

The internal storm structure is then generated as follows. Firstly, time intervals having zero rainfall are randomly assigned within the storm such that the sampled value of $p_{\mathrm{d}}$ is respected. It should be noted that the first and the last interval of the storm cannot have zero rainfall in order to preserve the duration $W$ of the storm. Furthermore, when the value of $p_{\mathrm{d}}$ is such that the storm should only contain one wet 10 min interval (i.e. $p_{\mathrm{d}}$ is close to 1 ), the rainfall depth is evenly divided among the first and last $10 \mathrm{~min}$ intervals. In addition, the total length of a dry spell within a storm is constrained to $23 \mathrm{~h}$, i.e. 1 hour less than the selection criterion, in order to avoid that one storm would result in two different storms when the same storm selection criterion is applied on the simulated rainfall series. It should also be mentioned that storms that have a duration smaller than $40 \mathrm{~min}$ and for which $p_{\mathrm{d}} \neq 0$, are disregarded in the generation of the rain- fall series, because of the inability to assure the generation of the imposed quartile storm.

Secondly, the cumulative storm depths are randomly selected. This procedure calculates the normalized cumulative depth at the end of a time interval, i.e. at time instant $b$, before moving to the next time interval. During the generation of the internal storm structure, three cases may occur, requiring different sampling strategies. These cases are:

1. Time instant $b$ is situated in between two consecutive wet 10 min intervals. In this case, a cumulative storm depth is randomly selected between the 10 and the $90 \%$ percentile curves, ensuring that the cumulative storm depths do not decrease in time. Figure 6 a illustrates this instant, where time instant $a$ denotes the previous time instant at which a value $D_{\mathrm{nc}}(a)$ was selected, with $D_{\text {nc }}$ the normalized cumulative storm depth. The current time instant $b$, at which a value $D_{\mathrm{nc}}(b)$ is to be selected, is also indicated. $D_{\mathrm{nc}}(b)$ is randomly selected between a minimal $\left(H_{10}(b)\right)$ and a maximal value $\left(H_{90}(b)\right)$, indicated in the figure. $H_{10}(b)$, respectively $H_{90}(b)$, denote the value of the $10 \%$, respectively $90 \%$ Huff curve, at instant $b$.

2. Time instant $b$ corresponds to the end of a dry period. In this case, depicted in Fig. 6b, no sampling has to be 
Table 3. Parameters of the bivariate Frank copulas in the construction of the 3- and 4-dimensional vine copulas.

\begin{tabular}{rrrr}
\hline & Parameter & $(W, V, D)$ & $\left(p_{\mathrm{d}}, W, V, D\right)$ \\
\hline & $\theta_{12}$ & - & 2.9632 \\
Season 1 & $\theta_{23}$ & 4.3567 & 8.9789 \\
& $\theta_{34}$ & -0.7276 & -0.6651 \\
& $\theta_{13 \mid 2}$ & - & -9.1797 \\
& $\theta_{24 \mid 3}$ & -0.4077 & 0.4199 \\
& $\theta_{14 \mid 23}$ & - & -0.4670 \\
\hline & $\theta_{12}$ & - & 3.4077 \\
Season 2 & $\theta_{23}$ & 6.3457 & 7.6379 \\
& $\theta_{34}$ & -0.6992 & -0.7037 \\
& $\theta_{13 \mid 2}$ & - & -.6601 \\
$\theta_{24 \mid 3}$ & 0.2150 & 0.2348 \\
& $\theta_{14 \mid 23}$ & - & -0.2741 \\
\hline & $\theta_{12}$ & - & 4.2445 \\
& $\theta_{23}$ & 6.1971 & 6.8144 \\
$\theta_{34}$ & 0.2221 & -0.7737 \\
Season 3 & $\theta_{13 \mid 2}$ & - & -6.0083 \\
& $\theta_{24 \mid 3}$ & -0.3429 & 0.0194 \\
& $\theta_{14 \mid 23}$ & - & -0.2419 \\
\hline & $\theta_{12}$ & - & 3.1574 \\
$\theta_{23}$ & 5.5352 & 8.0877 \\
$\theta_{34}$ & -0.9686 & -0.7041 \\
$\theta_{13 \mid 2}$ & - & -8.1955 \\
$\theta_{24 \mid 3}$ & -1.1409 & -0.0918 \\
& $\theta_{14 \mid 23}$ & - & -0.0958 \\
\hline & & &
\end{tabular}

performed (as the time interval between $a$ and $b$ should be dry), and thus the cumulative storm depth takes the value of the previous time instant, i.e. $D_{\mathrm{nc}}(b)=D_{\mathrm{nc}}(a)$, where $D_{\mathrm{nc}}(b)$ may be situated outside the percentile curves.

3. Time instant $b$ corresponds to the end of a wet period. In this third case, depicted in Fig. $6 \mathrm{c}$ and d, the dry period starts at time instant $b$ and ends at time instant $c$. Two sampling strategies are possible, among which is chosen with equal probability. It is allowed that a cumulative storm depth is sampled according to the 10 and $90 \%$ Huff curves either at time instant $b$ or at time instant $c$. When the first strategy is chosen (see Fig. 6c), $D_{\mathrm{nc}}(b)$ is sampled from the interval $\left[\max \left(D_{\mathrm{nc}}(a), H_{10}(b)\right), H_{90}(b)\right]$, and the sampled value can hence be smaller than $H_{10}(c)$, which indicates that the generated Huff curve will intersect the $10 \%$ Huff curve, before reaching time instant $c$ and will hence not remain between the 10 and $90 \%$ boundaries. When the second strategy is chosen (see Fig. $6 \mathrm{~d}$ ), $D_{\mathrm{nc}}(b)$ is drawn from $\left[\max \left(D_{\mathrm{nc}}(a), H_{10}(c)\right), H_{90}(c)\right]$, i.e. the sample is chosen according to the 10 and $90 \%$ Huff curves at time instant $c$. The sampled value can hence be larger than $H_{90}(b)$, which indicates that the generated Huff
Table 4. Probability of a storm to belong to a certain quartile group.

\begin{tabular}{lr}
\hline Quartile group & Probability [-] \\
\hline First & 0.3930 \\
Second & 0.2063 \\
Third & 0.1826 \\
Fourth & 0.2181 \\
\hline
\end{tabular}

curve will intersect the $90 \%$ Huff curve before reaching time instant $b$. Such flexibility is required as the fraction of dry spells often does not allow the curve to remain between the 10 and $90 \%$ boundaries. However, this flexibility is not a major problem, as at each time interval within the storm there are always $20 \%$ of the historical relative cumulative storm depths outside these boundaries by definition.

Based on the historical time series, it was observed that the increment in cumulative storm depth between two subsequent time instants in a Huff curve is not uniformly distributed (this observation was neglected in Vandenberghe et al., 2010a). Smaller increments occur more often than large increments. This behaviour is simulated by first establishing a cumulative probability distribution of strictly positive increments on the basis of the 105-year time series. To this end, for all storms in a particular season and quartile group, the frequencies of normalized (strictly positive) increments of cumulative rainfall depths between two subsequent wet periods were recorded. This empirical cumulative distribution function for the respective season and quartile group is then used to randomly select a normalized increase in storm depth for the subsequent wet time interval. Figure 7 illustrates the use of the cumulative distribution in the sampling procedure. In this figure, the minimum and maximum bounds of the increments are first determined on the basis of the Huff curves, as explained above. These bounds are then transferred to the cumulative distribution of normalized increments between which a value is randomly selected by uniformly sampling within this sampling range (see Fig. 7). The corresponding difference percentage of total storm depth is then obtained.

\section{Results}

It is common to validate the performance of a model through comparing statistics of one modelled time series to those calculated on the observed time series. However, given that the model has a stochastic nature, the statistics of the simulated time series will show some variability. To account for these stochastic effects, the model described in the previous section is employed to generate an ensemble of 100 time series of 105 years of $10 \mathrm{~min}$ rainfall (i.e. similar to the length of the observed time series). In order to evaluate whether the 


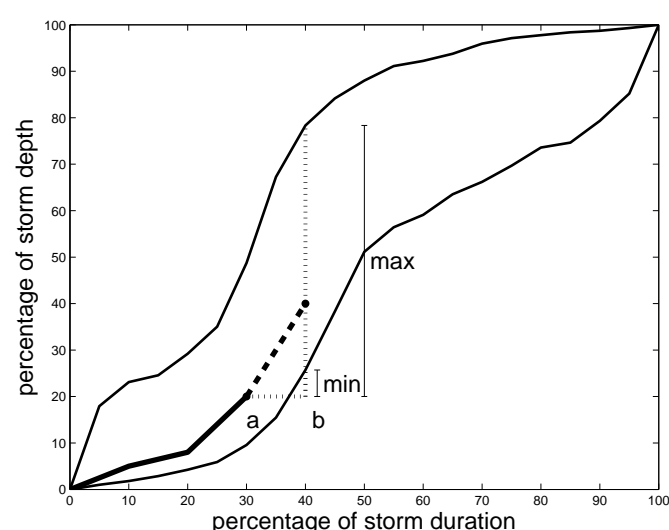

(a)

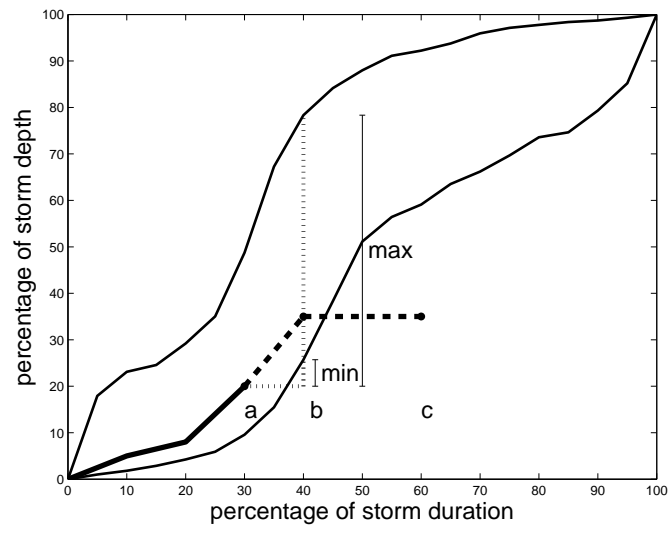

(c)

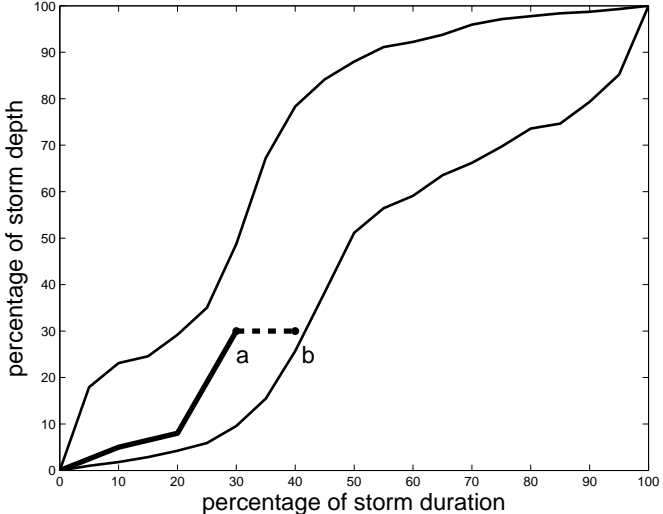

(b)

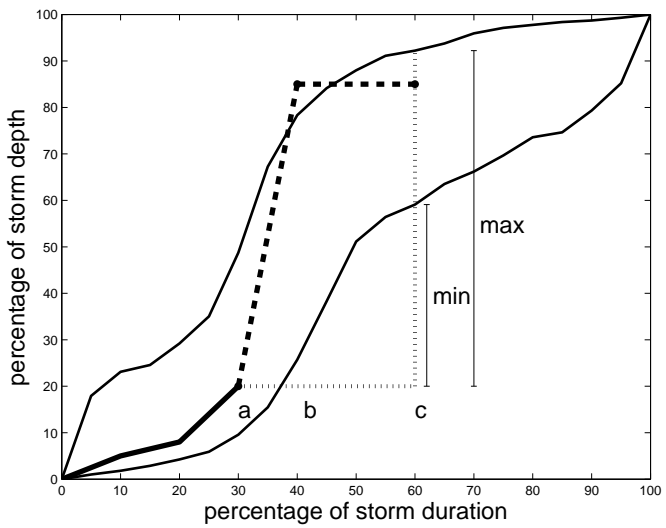

(d)

Figure 6. Illustration of the generation of an internal storm structure. The part of the Huff curve that is already generated (up to time instant a) is indicated by a thick solid line. The value at time instant $b$ needs to be determined. Four sampling strategies are possible: sampling in between two consecutive wet periods (case 1; a), sampling at the end of a dry period (case 2; b), sampling at the end of a wet period followed by a dry period with a selection on the basis of the current time instant (case 3; c) and with a selection on the basis of the last time instant in the dry period (case $3 ; \mathbf{d}$ ).

model performs well in the reproduction of aggregated rainfall statistics, the 100 time series are furthermore regarded as equally probable realizations and the statistics are calculated on a yearly basis. The traditional first- and second-order statistical moments (i.e. mean and variance), autocorrelation (AC) at different time lags and the zero depth probability (ZDP) are calculated along with the third-order central moment (skewness). These statistics are calculated on a yearly basis for each ensemble member at aggregation levels of $1 / 6$, $1,3,6,12$ and $24 \mathrm{~h}$. Thus, for an aggregation level, $100 \times 105$ values of each of these statistics are obtained, such that a bundle of 100 empirical cumulative distributions can be established, i.e. one distribution per ensemble member. The empirical cumulative distribution of the values of the statistics of the observed time series can then be compared with this bundle. If the empirical CDF of the observed statistics is situated within the bundle of distributions obtained by the model, the model performs well.
Figure 8a shows the bundles of the 100 empirical cumulative distributions of the yearly statistics of the time series generated by the copula-based model and the empirical cumulative distribution of the yearly statistics of the observed time series for a $10 \mathrm{~min}$ aggregation level. Figure $8 \mathrm{~b}$ displays the comparison for a $1 \mathrm{~h}$ aggregation level. These figures show that the observed mean is well represented by the copula-based model. For a 10 min aggregation level, the ZDP is also fairly well represented. The variance and the third central moment are overestimated, whereas the lag-1 and lag-2 autocovariances are underestimated. For the $1 \mathrm{~h}$ aggregation level, the ZDP and the lag-1 autocovariance are underestimated, the other statistics are fairly well represented. For the other aggregation levels, only the mean is well represented. The other statistics are sometimes well represented, underor overestimated. With respect to the ZDP statistic, the fact that this statistic is well represented at a $10 \mathrm{~min}$ aggregation level, yet underestimated at higher aggregation levels (except 

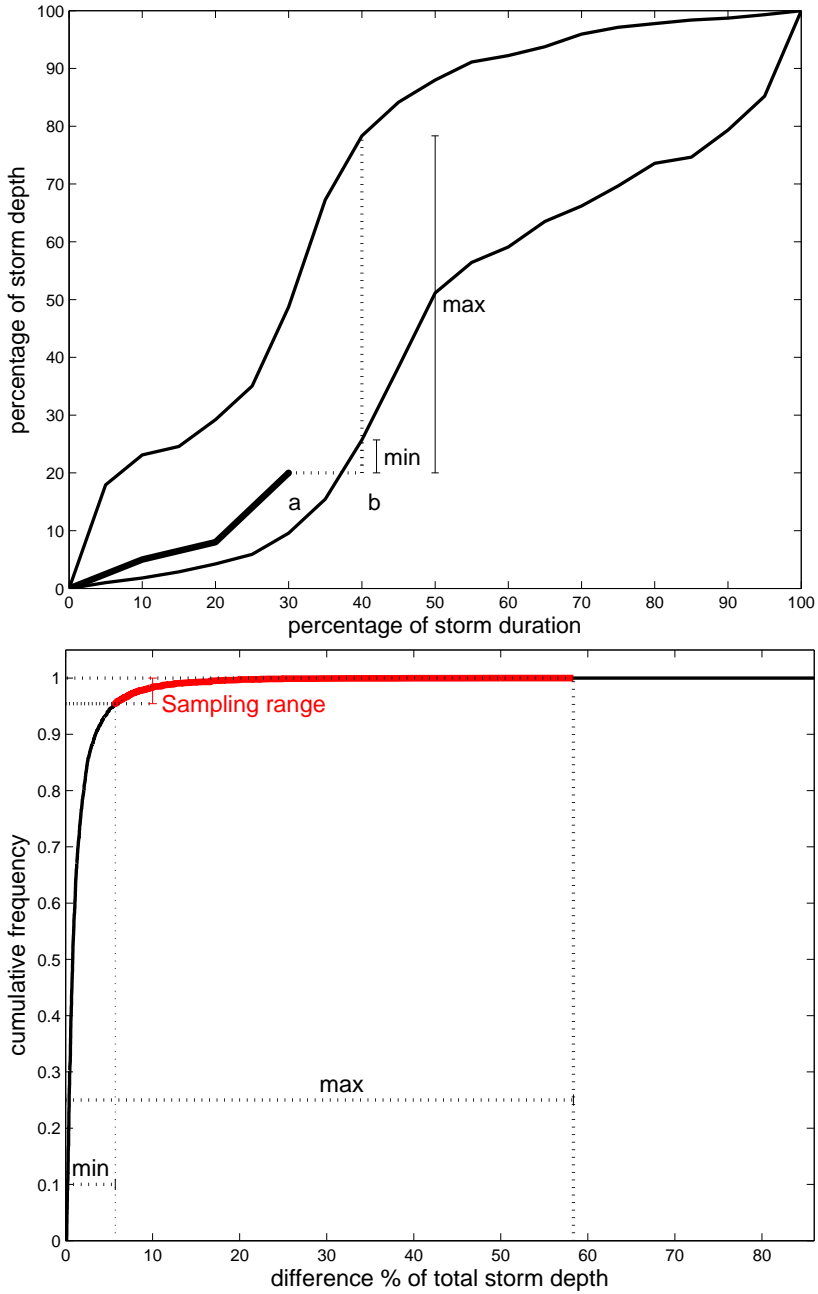

Figure 7. Illustration of the procedure to sample the storm depth at the next time instant (b). First, the minimal and maximal increment in percentage of storm depth at time instant $\mathrm{b}$ are determined (top panel). Then, the corresponding sampling range in the CDF of normalized increments is defined based on the minimal and maximal increment in percentage of storm depth derived from the top panel (bottom panel).

for a $24 \mathrm{~h}$ aggregation level), is probably due to the selection of the dry periods within the storm. For storms that have a duration of more than 1 hour, these zero intervals are probably not connected as no temporal correlation is taken into account during the selection of dry periods, such that fewer dry periods are obtained after the aggregation than what is observed in the Uccle time series. Future research will further elaborate on a better selection of dry periods within the storm.

As simulated time series are often used to simulate extreme discharges (Verhoest et al., 2010), the behaviour of the modelled extreme rainfall was also assessed. Figure 9 shows the annual maximum rainfall depths of the ensemble and of the observed rainfall series related to empirical
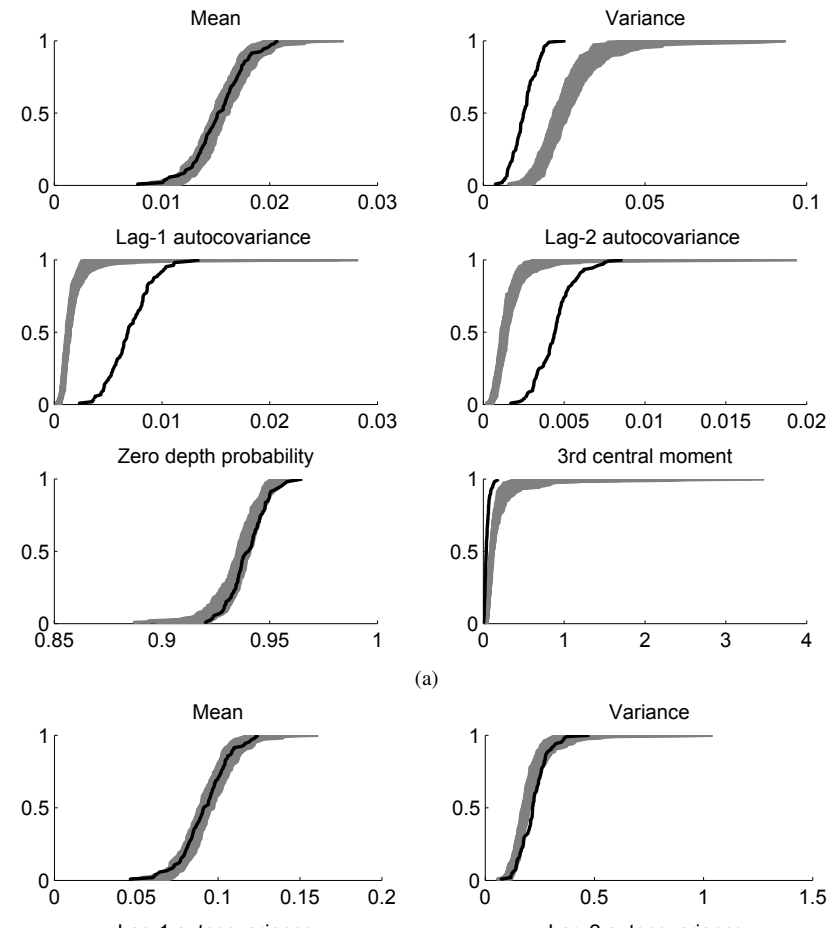

(a)
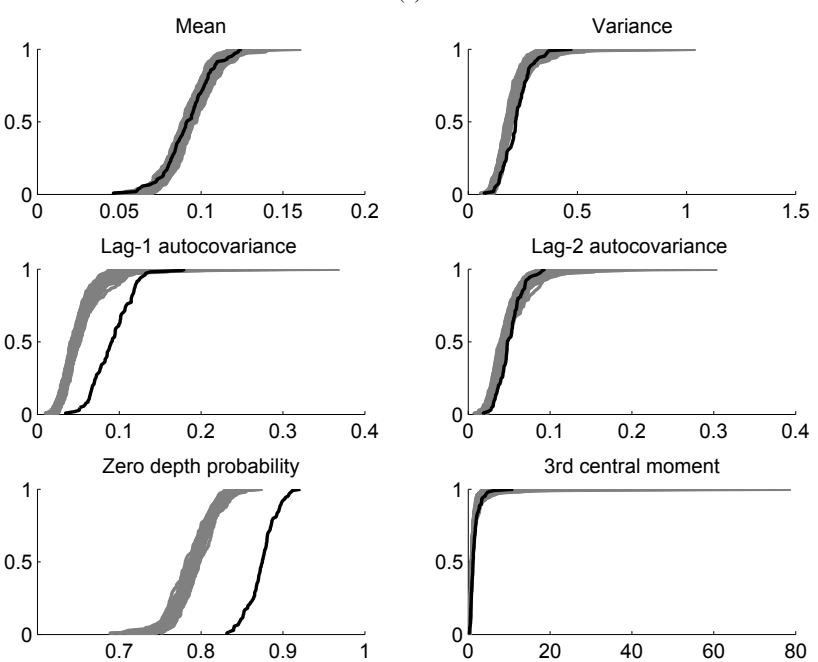

(b)

Figure 8. Comparison of the empirical cumulative distributions of the yearly statistics of the observed time series (black line) and the bundle of empirical cumulative distributions of synthetic time series generated by means of the copula-based model (grey) at a $10 \mathrm{~min}$ (a) and a $1 \mathrm{~h}$ aggregation level (b).

return periods, considering six different aggregation levels. This figure shows that the extrema are well modelled albeit the model overestimates extremes at short aggregation levels and tends to underestimate extremes at larger aggregation levels. Notwithstanding the shortcomings highlighted, this novel modelling concept holds promise. It should furthermore be stressed that other stochastic models such as the state-of-the-art Bartlett-Lewis models also are not able to preserve all statistics. 

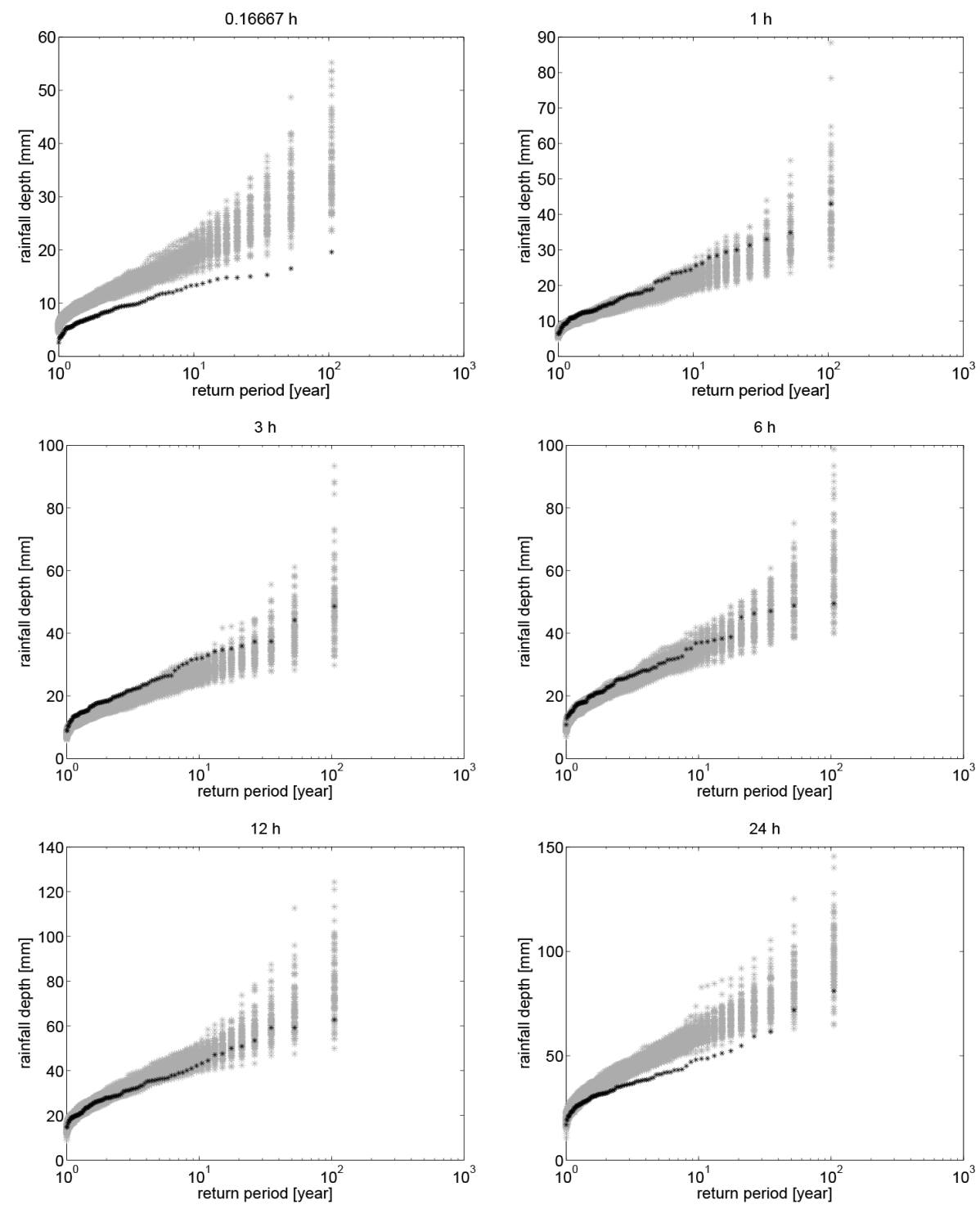

Figure 9. Comparison of empirically derived annual maxima related to the empirical return periods for different aggregation levels on the observed (black asterisks) and ensemble of synthetic time series generated by means of the copula-based rainfall model (grey asterisks).

\section{Conclusions}

This study is the first in its kind in which a continuous stochastic rainfall generator is developed that uses vine copulas to describe the storms and their arrival process. The internal storm structure is based on the concept of Huff curves, while the fraction of dry periods within the storm is determined by the copulas. The main advantage of this approach is that the model is completely data driven and is easier to calibrate than other rainfall generators such as the commonly used Modified Bartlett-Lewis model as, once the structure of the vine copula is determined, the calibration is reduced to estimating the parameters of the bivariate copulas. It should, however, be noted that we have at our disposal an exception- ally long time series of rainfall data on the basis of which the vine copulas are determined. If one would follow the same approach and search for the best-fitting copula family on a more commonly shorter time series of e.g. $<50$ years of rainfall data, one could be faced with difficulties as the number of storms per season may become too small for fitting the copulas.

The model applies 3- and 4-dimensional vine copulas to describe the dependence between the different storm characteristics. The 3-dimensional vine copulas are employed to describe the seasonal dependence between storm duration, storm volume and the interstorm period for storms that have no dry fraction within the storm. The 4-dimensional vine copulas are employed to describe the seasonal depen- 
dence between these storm characteristics and the dry fraction within the storm. These vine copulas were fitted to the observed storm characteristics of a 105-year time series of 10 min rainfall. Because of its frequent successful application in hydrological applications, the Frank copula family was chosen to be used within the vine copulas. On the basis of these vine copulas, values of these four storm characteristics were drawn, representing the external storm structure, ensuring a time series of 105 years of rectangular rainfall pulses. According to their seasonal probability of occurrence, storms with zero dry fractions were sampled from the 3-dimensional vine copulas. The internal storm structure of the rectangular pulses is superimposed based on Huff curves, which were identified on the basis of the observed time series, leading to the generation of continuous $10 \mathrm{~min}$ rainfall time series. In the generation of the internal storm structure, it is ensured that the fraction of dry periods within the storm as drawn from the vine copulas, is maintained. The internal storm structures are furthermore generated according to the probability of occurrence of the quartile storms in the observed time series, and the season in which they occur. In order to determine the difference of cumulative storm depths in the internal storm structure, the empirical cumulative PDF of increments between two subsequent wet periods in the storm is employed. In this way it is guaranteed that smaller increments occur more often than larger increments, as was observed in the measured time series.

In order to evaluate the performance of the rainfall model, an ensemble of 100 time series of ca. 105-year 10 min rainfall was generated, such that stochastic effects were accounted for. The results show that the copula-based rainfall model represents the mean value of the time series well, whereas the other statistics are either represented (fairly) well, overor underestimated, depending on the aggregation level. A second evaluation of the generated ensemble encompassed the calculation of the annual maximum series, for different aggregation levels. It was observed that the annual maxima simulated by means of the copula-based model were larger than the observed maxima for an aggregation level of $10 \mathrm{~min}$, and the moderate return period of the $24 \mathrm{~h}$ aggregation level. For aggregation levels of $1-12 \mathrm{~h}$ and the smaller and larger return periods of an aggregation level of $24 \mathrm{~h}$, a good correspondence between the simulated and observed extremes was observed. Future research will reveal whether the representation of the ZDP statistic for larger aggregation levels by the copula-based model can be improved by better selecting the internal dry storm periods. The performance of the copula-based model will also be compared to state-of-theart stochastic rainfall generators. Also, it should be investigated whether including other bivariate copula families in the vine copulas can further improve the performance of the vine-copula-based model.
Acknowledgements. This research has been performed in the framework of projects G.0837.10 and G.0013.11 granted by the Research Foundation Flanders.

Edited by: C. De Michele

\section{References}

Aas, K. and Berg, D.: Models for construction of multivariate dependence - a comparison study, Eur. J. Financ., 15, 639-659, 2009.

Aas, K., Czado, C., Frigessi, A., and Bakken, H.: Pair-copula constructions of multiple dependence, Insurance: Mathematics and Economics, 44, 182-198, 2009.

Bedford, T. and Cooke, R.: Probability density decomposition for conditionally dependent random variables modeled by vines, Ann. Math. Artif. Intel., 32, 245-268, 2001.

Bedford, T. and Cooke, R.: Vines - A new graphical model for dependent random variables, Ann. Stat., 30, 1031-1068, 2002.

Bonta, J. and Rao, A.: Factors affecting the identification of independent storm events, J. Hydrol., 98, 275-293, 1988.

Candela, A., Brigandì, G., and Aronica, G. T.: Estimation of synthetic flood design hydrographs using a distributed rainfallrunoff model coupled with a copula-based single storm rainfall generator, Nat. Hazards Earth Syst. Sci., 14, 1819-1833, doi:10.5194/nhess-14-1819-2014, 2014.

Chow, V., Maidment, D., and Mays, L.: Applied Hydrology, McGraw-Hill Book Company, New York, 588 pp., 1988.

De Jongh, I., Verhoest, N. E. C., and De Troch, F.: Analysis of a 105-year time series of precipitation observed at Uccle, Belgium, Internat. J. Climatol., 26, 2023-2039, 2006.

De Michele, C. and Salvadori, G.: A generalized Pareto intensityduration model of storm rainfall exploiting 2-copulas, J. Geophys. Res., 108, D24067, 2003.

De Michele, C., Salvadori, G., Canossi, M., Petaccia, A., and Rosso, R.: Bivariate statistical approach to check adequacy of dam spillway, J. Hydrol. Eng., 10, 50-57, 2005.

De Michele, C., Salvadori, G., Passoni, G., and Vezzoli, R.: A multivariate model of sea storms using copulas, Coast. Eng., 54, 734751, 2007.

Demarée, G.: The centennial recording raingauge of the Uccle Plateau: Its history, its data and its applications, La Houille Blanche - Revue International de l'Eau, 4, 95-102, 2003.

Evin, G. and Favre, A.-C.: A new rainfall model based on the Neyman-Scott process using cubic copulas, Water Resour. Res., 44, W03433, doi:10.1029/2007WR006054, 2008.

Evin, G. and Favre, A.-C.: Further developments of a transient Poisson-cluster model for rainfall, Stoch. Env. Res. Risk A., 27, 831-847, 2013.

Favre, A., Adlouni, S. E., Perreault, L., Thiemonge, N., and Bobee, B.: Multivariate hydrological frequency analysis using copulas, Water Resour. Res., 40, W01101, doi:10.1029/2003WR002456, 2004.

Ganguli, P. and Reddy, M.: Probabilistic assessment of flood risks using trivariate copulas, Theor. Appl. Climatol., 111, 341-360, 2013.

Genest, C.: Frank's family of bivariate distributions, Biometrika, 74, 549-555, 1987. 
Genest, C., Favre, A., Beliveau, J., and Jacques, C.: Metaelliptical copulas and their use in frequency analysis of multivariate hydrological data, Water Resour. Res., 43, W09401, doi:10.1029/2006WR005275, 2007.

Gräler, B.: Modelling skewed spatial random fields through the spatial vine copula, Spatial Statistics, 10, 87-102, 2014.

Gräler, B., van den Berg, M. J., Vandenberghe, S., Petroselli, A., Grimaldi, S., De Baets, B., and Verhoest, N. E. C.: Multivariate return periods in hydrology: a critical and practical review focusing on synthetic design hydrograph estimation, Hydrol. Earth Syst. Sci., 17, 1281-1296, doi:10.5194/hess-17-12812013, 2013.

Grimaldi, S. and Serinaldi, F.: Design hyetograph analysis with 3copula function, Hydrolog. Sci. J., 51, 223-238, 2006.

Gyasi-Agyei, Y.: Copula-based daily rainfall disaggregation model, Water Resour. Res., 47, W07535, doi:10.1029/2011WR010519, 2011.

Gyasi-Agyei, Y. and Melching, C.: Modelling the dependence and internal structure of storm evens for continuous rainfall simulation, J. Hydrol., 464, 249-261, 2012.

Hobæk Haff, I., Aas, K., and Frigessi, A.: On the simplified paircopula construction - Simply useful or too simplistic?, J. Multivariate Anal., 101, 1296-1310, 2010.

Huff, F.: Time distribution of rainfall in heavy storms, Water Resour. Res., 3, 1007-1019, 1967.

Joe, H.: Multivariate Models and Dependence Concepts, Chapman and Hall, 399 pp., 1997.

Kao, S. and Govindaraju, R.: Trivariate statistical analysis of extreme rainfall events via the Plackett family of copulas, Water Resour. Res., 44, W02415, doi:10.1029/2007WR006261, 2008.

Katz, R. and Parlange, M.: Overdispersion phenomenon in stochastic modeling of precipitation, J. Climate, 11, 591-601, 1998.

Kavvas, M. and Delleur, J.: A stochastic cluster model of daily rainfall sequences, Water Resour. Res., 17, 1151-1160, 1981.

Kurowicka, D. and Cooke, R.: Sampling algorithms for generating joint uniform distributions using the vine-copula method, Comput. Stat. Data An., 51, 2889-2906, 2007.

Ma, M., Song, S., Ren, L., Jiand, S., and Song, J.: Multivariate drought characteristics using trivariate Gaussian and Student $\mathrm{t}$ copulas, Hydrol. Process., 27, 1175-1190, 2013.

Menabde, M. and Sivapalan, M.: Modeling of rainfall time series and extremes using bounded random cascades and Levy-stable distributions, Water Resour. Res., 36, 3293-3300, 2000.

Mendes, B. and Accioly, V.: Robust Pair-Copula based forecasts of realized volatitiliy, Applied Stochastic Models in Business and Industry, 30, 183-199, 2014.

Nelsen, R.: An Introduction to Copulas, in: Lecture Notes in Statistics, edited by: Bickel, P., Diggle, P., Fienberg, S., Krickeberg, K., Olking, I., Wermuth, N., and Zeger, S., pp. 216, Springer-Verlag, New York, 2006.

Nikololoupoulos, A., Joe, H., and Li, H.: Vine coplas with asymmetric tail dependence and applications to financial return data, Computational Statistics and Data Analysis, 56, 3659-3673, 2012.

Ntegeka, V. and Willems, P.: Trends in multidecadal oscillations in rainfall extremes, based on a more than 100-year time series of 10 min rainfall intensities at Uccle, Belgium, Water Resour. Res., 44, W07402, doi:10.1029/2007WR006471, 2008.
Onof, C., Chandler, R., Kakou, A., Northrop, P., Wheater, H., and Isham, V.: Rainfall modelling using Poisson-cluster processes: a review of developments., Stoch. Env. Res. Risk A., 14, 384-411, 2000.

Pan, M., Yuan, X., and Wood, E. F.: A probabilistic view on drought recovery., Geophys. Res. Lett., 40, 3637-3642, 2013.

Pham, M. T., Vanhaute, W. J., Vandenberghe, S., De Baets, B., and Verhoest, N. E. C.: An assessment of the ability of BartlettLewis type of rainfall models to reproduce drought statistics, Hydrol. Earth Syst. Sci., 17, 5167-5183, doi:10.5194/hess-17-51672013, 2013.

Restrepo-Posada, P. and Eagleson, P.: Identification of independent rainstorms, J. Hydrol., 55, 303-319, 1982.

Rodriguez-Iturbe, I., Cox, D., and Isham, V.: Some models for rainfall based on stochastic point processes, Proc. R. Soc. Lon. Ser.A, 410, 269-288, 1987a.

Rodriguez-Iturbe, I., Febres de Power, B., and Valdés, J.: Rectangular pulses point process models for rainfall: analysis of empirical data, . Geophys. Res., 92, 9645-9656, 1987b.

Salvadori, G. and De Michele, C.: Analytical calculation of storm volume statistics involving Pareto-like intensityduration marginals, Geophys. Res. Lett., 31, L04502, doi:10.1029/2003GL018767, 2004.

Salvadori, G. and De Michele, C.: Statistical characterization of temporal structure of storms, Adv. Water Resour., 29, 827-842, 2006.

Salvadori, G. and De Michele, C.: On the use of copulas in hydrology: theory and practice, J. Hydrol. Eng., 12, 369-380, 2007.

Serinaldi, F. and Grimaldi, S.: Fully nested 3-copula: procedure and application on hydrological data, J. Hydrol. Eng., 12, 420-430, 2007.

Sklar, A.: Fonctions de répartition á $n$ dimensions et leurs marges., Publications de l'Institut de Statistique de l'Université de Paris, 8, 229-231, 1959.

Song, S. and Singh, V.: Meta-elliptical copulas for drought frequency analysis of periodic hydrologic data, Stoch. Env. Res. Risk A., 24, 425-444, 2010.

Vaes, G., Willems, P., and Berlamont, J.: 100 years of Belgian rainfall: Are there trends?, Water Sci. Technol., 45, 55-61, 2002.

Vandenberghe, S., Verhoest, N. E. C., Buyse, E., and De Baets, B.: A stochastic design rainfall generator based on copulas and mass curves, Hydrol. Earth Syst. Sci., 14, 2429-2442, doi:10.5194/hess-14-2429-2010, 2010a.

Vandenberghe, S., Verhoest, N. E. C., and De Baets, B.: Fitting bivariate copulas to the dependence structure between storm characteristics: A detailed analysis based on 105 year 10 min rainfall, Water Resour. Res., 46, W01512, doi:10.1029/2009WR007857, 2010b.

Vandenberghe, S., Verhoest, N. E. C., Onof, C., and De Baets, B.: A comparative copula-based bivariated frequency analysis of observed and simulated storm evens: a cast study on BartlettLewis modeled rainfall, Water Resour. Resear., 47, W07529, doi:10.1029/2009WR008388, 2011.

Vanhaute, W. J., Vandenberghe, S., Scheerlinck, K., De Baets, B., and Verhoest, N. E. C.: Calibration of the modified BartlettLewis model using global optimization techniques and alternative objective functions, Hydrol. Earth Syst. Sci., 16, 873-891, doi:10.5194/hess-16-873-2012, 2012. 
Verhoest, N., Troch, P., and De Troch, F.: On the applicability of Bartlett-Lewis rectangular pulses models in the modeling of design storms at a point, J. Hydrol., 202, 108-120, 1997.

Verhoest, N. E. C., Vandenberghe, S., Cabus, P., Onof, C., MecaFigueras, T., and Jameleddine, S.: Are stochastic point rainfall models able to preserve extreme flood statistics?, Hydrol. Process., 24, 3439-2445, 2010.

Viglione, A., Castellarin, A., Rogger, M., Merz, R., and Blöschl, G.: Extreme rainstorms: Comparing regional envelope curves to stochastically generated events, Water Resour. Res., 48, W01509, doi:10.1029/2011WR010515, 2012.

Willems, P.: A spatial rainfall generator for small spatial scales, J. Hydrol., 252, 126-144, 2001.
Wong, G., Lambert, M., Leonard, M., and Metcalfe, A.: Drought Analysis using trivariate copulas conditional on climatic states, J. Hydrol. Eng., 15, 129-141, 2010.

Xiong, L., Yu, K., and Gottschalk, L.: Estimation of the distribution of annual runoff from climatic variables using copulas, Water Resour. Res., 50, 7134-7152, 2014.

Zhang, D.: Vine copulas and applications to the European Union sovereign debt analysis, International Review of Financial Analysis, 36, 46-56, 2014.

Zhang, L. and Singh, V.: Gumbel-Hougaard copula for trivariate rainfall frequency analysis, J. Hydrol. Eng., 12, 409-419, 2007. 